\title{
A clinical nomogram for predicting tumor regression grade in esophageal squamous-cell carcinoma treated with immune neoadjuvant immunotherapy
}

\author{
Yongkui Yu ${ }^{1}$, Wei Wang ${ }^{1}$, Zimin Qin ${ }^{1}$, Haomiao Li $^{1}$, Qi Liu ${ }^{1}$, Haibo Ma ${ }^{1}$, Haibo Sun ${ }^{1}$, Thomas L. Bauer ${ }^{2}$, \\ Jose M. Pimiento ${ }^{3}$, Emmanuel Gabriel ${ }^{4}$, Thomas Birdas ${ }^{5}$, Yin $\mathrm{Li}^{6}$, Wenqun Xing ${ }^{1}$ \\ ${ }^{1}$ Department of Thoracic Surgery, The Affiliated Cancer Hospital of Zhengzhou University/Henan Cancer Hospital, Zhengzhou, China; \\ ${ }^{2}$ Department of Surgery, Jersey Shore University Medical Center, Department of General Surgery, Hackensack Meridian School of Medicine, Nutley, \\ NJ, USA; ${ }^{3}$ Department of Gastrointestinal Oncology, Moffitt Cancer Center and Research Institute, Tampa, FL, USA; ${ }^{4}$ Department of Surgery, \\ Mayo Clinic, Jacksonville, FL, USA; ${ }^{5}$ Department of Surgery, Thoracic Division, Indiana University Melvin and Bren Simon Comprehensive Cancer \\ Center, Indianapolis, IN, USA; ${ }^{\circ}$ Department of Thoracic Surgery, Cancer Hospital, Chinese Academy of Medical Sciences, Beijing, China \\ Contributions: (I) Conception and design: Y Yu, Y Li, W Xing; (II) Administrative support: Y Yu, Y Li, W Xing; (III) Provision of study materials or \\ patients: Y Yu, W Wang, Z Qin, H Li, Q Liu, H Ma, H Sun; (IV) Collection and assembly of data: Y Yu, W Wang, Z Qin, H Li, Q Liu, H Ma, H \\ Sun; (V) Data analysis and interpretation: Y Yu, H Ma, H Sun; (VI) Manuscript writing: All authors; (VII) Final approval of manuscript: All authors. \\ Correspondence to: Yin Li. Department of Thoracic Surgery, Cancer Hospital, Chinese Academy of Medical Sciences, No. 17, Panjiayuan \\ South Lane, Chaoyang District, Beijing 100021, China. Email: $513123607 @ q q . c o m ;$ Wenqun Xing. Department of Thoracic Surgery, \\ The Affiliated Cancer Hospital of Zhengzhou University/Henan Cancer Hospital, No. 127, Dongming Road, Zhengzhou 450008, China. \\ Email: xingwenqun@sina.com.
}

Background: There are various treatment options for esophageal squamous cell cancer. including surgery, peri-operative chemotherapy, and radiation. More recently, neoadjuvant immunotherapy has also been shown improve outcomes. In this study, we addressed the question, "Can we predict which patients with esophageal squamous cell cancer will benefit from neoadjuvant immunotherapy?”.

Methods: All patients with thoracic esophageal squamous-cell carcinoma (T2N+M0-T3-4N0/+M0) (according to the eighth edition of the National Comprehensive Cancer Network guidelines) who underwent immune neoadjuvant immunochemotherapy with programmed cell death protein 1 (PD-1) combined with paclitaxel plus cisplatin or nedaplatin in the Affiliated Cancer Hospital of Zhengzhou University, China, between November 2019 and August 2021 were included in this study. All patients underwent surgical resection. We developed a response [tumor regression grade (TRG)] prediction model using the least absolute shrinkage and selection operator (LASSO) regression incorporating factors associated with response. The accuracy of the prediction model was then validated.

Results: We included 79 patients who underwent neoadjuvant immunotherapy combined with chemotherapy, aged $48-78$ years $(62.05 \pm 6.67)$, including 21 males and 58 females. There were five cases of immune-related pneumonia, of which three cases were diagnosed as immune-related pneumonia during the perioperative period, and one case of immune-related thyroid dysfunction changes. After LASSO regression, the factors that were independently associated with TRG were clinical T stage before neoadjuvant therapy, clinical $\mathrm{N}$ stage before neoadjuvant therapy, albumin level difference from before to after neoadjuvant therapy, white blood cell (WBC) count before neoadjuvant therapy, and T stage before surgery. We constructed a prediction model, plotted the nomogram, and verified its accuracy. Its Brier score was 0.13 , its calibration slope was 0.98, and its C-index was 0.90 (95\% CI: 0.82-0.97).

Conclusions: Our prediction model can predict the likelihood of TRG in patients with esophageal squamous cell cancer after immunotherapy combined with neoadjuvant chemotherapy. Using this prediction model, we plan to conduct a subsequent neoadjuvant radiotherapy in patients with of TRG 2-3 patients with neoadjuvant radiotherapy. 
Keywords: Esophageal squamous cell cancer; immunotherapy; pathological remission grading; prediction model

Submitted Nov 04, 2021. Accepted for publication Jan 20, 2022.

doi: 10.21037/atm-22-78

View this article at: https://dx.doi.org/10.21037/atm-22-78

\section{Introduction}

Risk factors for the development of esophageal squamous cell carcinoma include low socioeconomic status, consumption of tobacco, alcohol, hot beverages, and nitrosamines (1). There are different neoadjuvant treatment options for esophageal cancer, with a wide range of clinical studies such as NEOCRTEC5010 and CROSS have shown that for patients with locally advanced esophageal cancer, neoadjuvant chemoradiotherapy can improve the short term and long-term outcomes (2-6). However, the CROSS trial had an R0 resection rate in the surgeryonly group of $69 \%$, which was significantly lower than the $92 \%$ in the chemoradiotherapy + surgery group $(\mathrm{P}<0.001)$. Whether R0 resection was an important factor affecting the prognosis is not clear. The FFCD9901 study showed that neoadjuvant chemoradiotherapy not only did not increase the $\mathrm{R} 0$ resection rate but did increase the postoperative mortality rate, and the study was eventually discontinued (7). In a metanalysis in 2018 neoadjuvant chemoradiotherapy seemed to increase postoperative mortality (RR 1.46 and 1.58 , respectively) (8). The Neoadjuvant Chemotherapy Versus Chemoradiotherapy for Cancer of the Esophagus or Cardia (NeoRes I) study, the study of Klevebro et al., and a meta-analysis all showed that neoadjuvant chemoradiotherapy did not boost survival compared with neoadjuvant chemotherapy (9-11). The RTOG Trial 8911 showed that neoadjuvant chemotherapy did not improve the survival of patients (12). Other studies have shown that neoadjuvant chemotherapy can benefit patients in terms of survival compared to surgery alone $(13,14)$, while neoadjuvant chemoradiotherapy may not necessarily benefit patients (15). While neoadjuvant chemoradiotherapy and neoadjuvant chemotherapy alone can benefit patients, in this study we addressed the question, "Can emerging immunotherapy technologies also benefit the survival of esophageal squamous cell cancer patients?".

Programmed cell death protein 1/programmed cell death ligand 1 (PD-1/PD-L1) inhibitors can activate $\mathrm{T}$ lymphocytes, inhibit tumor growth, and improve the prognosis of tumor patients. Many PD-1/PD-L1 inhibitors can treat melanoma, lung cancer, and renal cell cancer (16-18). There are many clinical studies on the use of PD-1/PD-L1 inhibitors in immunotherapies for esophageal cancer (19). A phase 2 trial, KEYNOTE-180, showed that the objective response rate (ORR) of pembrolizumab monotherapy was $14.3 \%$ (20). A phase 3 trial, KEYNOTE-181, showed that pembrolizumab for the treatment of advanced esophageal squamous-cell cancer patients with PD-L1 combined positive score $\geq 10$ had a significantly longer median survival (8.2 vs $7.1 \mathrm{~m}, \mathrm{P}=0.0095)$ and an ORR of $21.5 \%$ (20). PD-1/PD-L1 inhibitors have proven safety and efficacy in the treatment of esophageal squamous-cell carcinoma.

The better the tumor regression grade (TRG) after neoadjuvant therapy, the higher the 5 -year survival rate (21-23). Patients with TRG $0-1$ after neoadjuvant chemotherapy combined with immunotherapy could have a theoretically better prognosis than patients with TRG 2-3. We believe that for patients with TRG 2-3, neoadjuvant radiotherapy may also be added to increase the chance of better response. One study also investigated clinical factors and treatment which have prognostic evaluation of esophageal cancer (24), but not like us, if our study could screen out patients with TRG 2-3, radiation therapy was added next, it could benefit these patients in terms of survival, and ultimately all patients benefit from different treatment modes. To determine whether we could predict the benefit of neoadjuvant immunochemotherapy for esophageal cancer, we developed a prediction model for TRG.

We present the following article in accordance with the TRIPOD reporting checklist (available at https://atm. amegroups.com/article/view/10.21037/atm-22-78/rc).

\section{Methods}

All patients with thoracic esophageal squamous-cell carcinoma (T2N+M0-T3-4N0/+M0) (according to the eighth edition of the National Comprehensive Cancer Network guidelines) who underwent neoadjuvant chemotherapy with PD-1 combined with paclitaxel plus cisplatin or nedaplatin at The Affiliated Cancer Hospital 
of Zhengzhou University, China, between November 2019 and August 2021 were included. The study was conducted in accordance with the Declaration of Helsinki (as revised in 2013). This study was approved by the Ethics Committee of The Affiliated Cancer Hospital of Zhengzhou University (ethics number 2019092702), and informed consent was taken from all individual participants. Prior to surgery, enhanced computed tomography (CT) or enhanced magnetic resonance imaging of the chest and upper abdomen, cardiac ultrasound, abdominal color Doppler ultrasound, upper gastrointestinal tract contrast, ultrasound gastroscopy, pulmonary function, and laboratory tests were performed. Before neoadjuvant therapy, PD-L1 expression was detected in esophagogastroscopic biopsy specimens by immunohistochemistry.

Prior to surgery, patients received two cycles of paclitaxel $\left(135-175 \mathrm{mg} / \mathrm{m}^{2}\right)$, cisplatin $\left(80-120 \mathrm{mg} / \mathrm{m}^{2}\right.$, D1 or D1-4), or nedaplatin $\left(80-100 \mathrm{mg} / \mathrm{m}^{2}\right.$, D1 or D1-4), as well as PD-L1 inhibitor $\left(200 \mathrm{mg} / \mathrm{m}^{2}\right)$. The surgical approaches included open or laparo-thoracoscopic McKeown surgery. PD-1 monoclonal antibodies for immunotherapy included tislelizumab, camrelizumab (Hengrui, Lianyungang, Jiangsu), toripalimab (Junshi Biotechnology, Pudong, Shanghai), Keytruda (Merck, New Jersey, USA), and sintilimab (Innovent Bio, Suzhou, Jiangsu).

\section{Development of the prediction model}

We referred to the Ryan scoring system to assign TRGs (25). The TRG 0-3 are these: 0: no viable cancer cells (complete response); 1: single cell or rare small groups of cancer cells (near complete response); 2: residual cancer with evident tumor regression but more than single cell or rare small groups of cancer cells (partial response); 3: extensive residual cancer with no evident tumor regression (poor or not response). Three pathologists were asked to reexamine the results of the pathological sections, and the final TRG grade had to be agreed upon by two or more pathologists. We divided the postoperative TRGs into two categories: grades 0 and 1 were combined into one category, and grades 2 and 3 were combined into another category. We abstracted and categorized the following demographic and tumor variables: age, sex, hypertension, diabetes, other comorbidities, smoking history, alcohol history, type of PD-1 pulmonary comorbidities, $\mathrm{T}$ stage before treatment, $\mathrm{N}$ stage, body mass index (BMI), nutritional score, white blood cell (WBC) count, hemoglobin, lymphocyte count, monocyte count, albumin, bilirubin, cholesterol, $\mathrm{T}$ stage after chemotherapy, changes in
BMI, WBC count, hemoglobin, lymphocyte count, monocyte count, albumin, bilirubin, and cholesterol from before to after chemotherapy; and whether PD-1 was expressed.

The predictive accuracy of the model was assessed using three measures: (I) Brier score for overall performance; (II) calibration slope for calibration; and (III) concordance index (C-index) for discrimination. In addition to these numeric measures, we used the calibration plot and receiver operating characteristic curve to display the calibration and discrimination aspects of our final model. The closer the Brier score is to 0 , the better the predictive ability, and the closer the standard slope is to 1 , the closer the predicted value is to the result. A C-index closer to 1 indicated better discrimination. These measures were used together to evaluate the accuracy of the model's predictions. To this end, we conducted multiple repeated evaluation and took the model with the highest prediction accuracy as the final model.

\section{Creation of the nomogram}

The final results are presented as a nomogram. The nomogram contains a reference line on the top for scoring the points of each predictor from 0 to 100 . The predictive variables are displayed below with bars that scale their effect size, visually demonstrating visually the relative weight of each variable and allowing for points to be assigned to each significant clinical characteristic (26). The overall score of each predictive factor and the corresponding probability of the occurrence of TRG 0-1 can be read on the second line from the bottom. We also plotted the decision curve and clinical impact curve to validate the prediction model.

\section{Statistical analysis}

All statistical analyses were performed using R 3.63 (https:// www.r-project.org/). The best predictors of TRG were screened by the least absolute shrinkage and selection operator (LASSO) regression using the "glmnet" package in R. The "rms" package was used to incorporate the factors selected by LASSO regression into the multivariate logistic regression analysis to build a prediction model.

\section{Results}

\section{Baseline clinical characteristics}

We enrolled 79 patients who underwent neoadjuvant 
Table 1 Patient and tumor characteristics of this study

\begin{tabular}{|c|c|}
\hline Variables & Overall cohort $(n=79)$ \\
\hline Age (years) & $62.05 \pm 6.67$ \\
\hline Sex (males/females) & $58(73.4 \%) / 21(26.6 \%)$ \\
\hline cT stage before INJT (1/2/3/4) & $0 / 12 / 55 / 12$ \\
\hline cN stage before INJT (-/+) & $27(34.2 \%) / 52(65.8 \%)$ \\
\hline cT stage after INJT (1/2/3/4) & $38 / 25 / 15 / 1$ \\
\hline cN stage after INJT (-/+) & $51(64.6 \%) / 28(35.4 \%)$ \\
\hline pT $(0 / 1 / 2 / 3 / 4)$ & $26 / 11 / 14 / 28 / 0$ \\
\hline $\mathrm{pN}(0 / 1 / 2 / 3)$ & $59 / 15 / 5 / 0$ \\
\hline cTNM stage (I/II/III/IVa) & $0 / 3 / 74 / 2$ \\
\hline pTNM stage (I/II/III/IVa) & $42 / 17 / 8 / 12$ \\
\hline Hypertension (yes/no) & $16(20.3 \%) / 63(79.7 \%)$ \\
\hline Diabetes (yes/no) & $4(5.1 \%) / 75$ (94.9\%) \\
\hline Smoking (yes/no) & $50(63.3 \%) / 29(36.7 \%)$ \\
\hline Drinking (yes/no) & $43(54.4 \%) / 36(45.6 \%)$ \\
\hline Location $(\mathrm{u} / \mathrm{m} / \mathrm{l})$ & $13 / 53 / 13$ \\
\hline Surgery (open/VATS) & $14(17.7 \%) / 65(82.3 \%)$ \\
\hline Tumor regression grade $(0 / 1 / 2 / 3)$ & $25 / 12 / 28 / 14$ \\
\hline Tumor regression grade $(0-1 / 2-3)$ & $37(46.8 \%) / 42(53.2 \%)$ \\
\hline WBCs before INJT $\left(\times 10^{9} / \mathrm{L}\right)$ & $6.37 \pm 1.92$ \\
\hline BMI before INJT $\left(\mathrm{kg} / \mathrm{m}^{2}\right)$ & $22.96 \pm 2.83$ \\
\hline $\mathrm{Hb}$ before INJT (g/L) & $136.24 \pm 17.49$ \\
\hline Albumin before INJT (g/L) & $41.61 \pm 3.31$ \\
\hline Changes in albumin & $0.77 \pm 4.34^{*}$ \\
\hline WBCs before surgery $\left(\times 10^{9} / \mathrm{L}\right)$ & $6.28 \pm 2.05$ \\
\hline BMI before surgery $\left(\mathrm{kg} / \mathrm{m}^{2}\right)$ & $22.30 \pm 7.18$ \\
\hline $\mathrm{Hb}$ before surgery $(\mathrm{g} / \mathrm{L})$ & $122.16 \pm 14.42$ \\
\hline Albumin before surgery $(\mathrm{g} / \mathrm{L})$ & $40.81 \pm 2.99$ \\
\hline Duration of surgery (min) & $292.69 \pm 92.02$ \\
\hline \multicolumn{2}{|l|}{ PD-1, n (\%) } \\
\hline Camrelizumab & $14(17.7)$ \\
\hline Keytruda & $1(1.3)$ \\
\hline Toripalimab & $57(72.2)$ \\
\hline Sintilimab & $5(6.3)$ \\
\hline Tislelizumab & $2(2.5)$ \\
\hline Immune pneumonia (yes/no) & $5(6.3 \%) / 74(93.7 \%)$ \\
\hline Changes in thyroid function (yes/no) & $1(1.3 \%) / 78$ (98.7\%) \\
\hline
\end{tabular}

*, if the absolute value of the change is calculated, it should be $3.24 \pm 3.00$. INJT, immune neoadjuvant therapy; u/m/l, upper/ middle/lower; VATS, video-assisted thoracoscopic surgery; BMI, body mass index; WBC, white blood cell; PD-1, programmed cell death protein 1 . immunotherapy combined with chemotherapy. They were aged $48-78$ years $(62.05 \pm 6.67$ years $)$, and there were 58 males and 21 females. There were five cases of immunerelated pneumonia, of which three cases were diagnosed as immune-related pneumonia during the perioperative period and two cases were diagnosed as immune-related pneumonia during continued immunotherapy after surgery. Immune-related thyroid function changes occurred in one case, which occurred during postoperative immune maintenance therapy. Patient demographic and tumor characteristics are listed in Table 1.

After LASSO regression analysis, the factors that were independently associated with the TRG included clinical $\mathrm{T}$ stage before neoadjuvant therapy, clinical $\mathrm{N}$ stage before neoadjuvant therapy, albumin difference before $v$ s. after neoadjuvant therapy, WBC count before neoadjuvant therapy, and preoperative T stage (Figure 1A,1B, Table 2). The coefficient $\lambda$ decreased with a greater number of variables. When $\lambda$ was optimal, the coefficients of the excluded variables were compressed to 0 , while the coefficients of the variables left in the model were nonzero. The results show that the optimal value of $\lambda$ was 0.080700 , and $\ln (\lambda)=-2.517017$ (Figure 1). Through the LASSO analysis, the 38 clinically relevant factors that were initially inputted were reduced to five potential predictors (Table 2).

Since the $\mathrm{P}$ value of $\mathrm{cN}$ was less than 0.05 , we created another regression model that excluded $\mathrm{cN}$ and then compared the Akaike's information criterion (AIC) values of the two regression models. The results showed that the AIC value of the regression equation was 86.5 with $\mathrm{cN}$ included vs. 87.7 after removing $\mathrm{cN}$. Thus, we finally choose to include $\mathrm{cN}$ in the regression equation.

Finally, we present the prediction model as a nomogram (Figure 2A). We analyzed the degree of TRG variance associated with each factor, and the results showed that $\mathrm{cT}$ stage after immune neoadjuvant therapy (INJT) and change in albumin contributed the most to the TRG variance (Figure 2B).

The accuracy of this prediction model was then verified: The Brier score was 0.13, the calibration slope was 0.98 , and the C-index was 0.90 (95\% CI: 0.82-0.97). The calibration slope and the receiver operating characteristic curve were also plotted to graphically assess calibration and discrimination, respectively (Figure $3 A$ ). The calibration slope tests the concordance between predicted values and outcomes with a perfect slope equal to 1 . The C-index tests the discrimination of the model, or the ability to tell which patients who have TRG 2-3 should be given neoadjuvant 
A

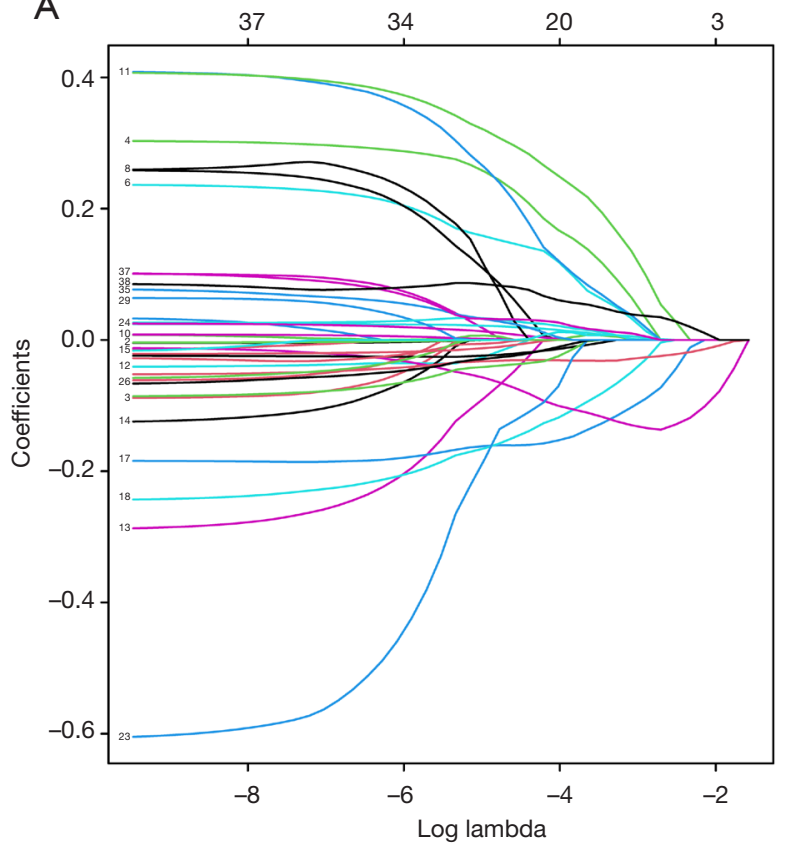

B

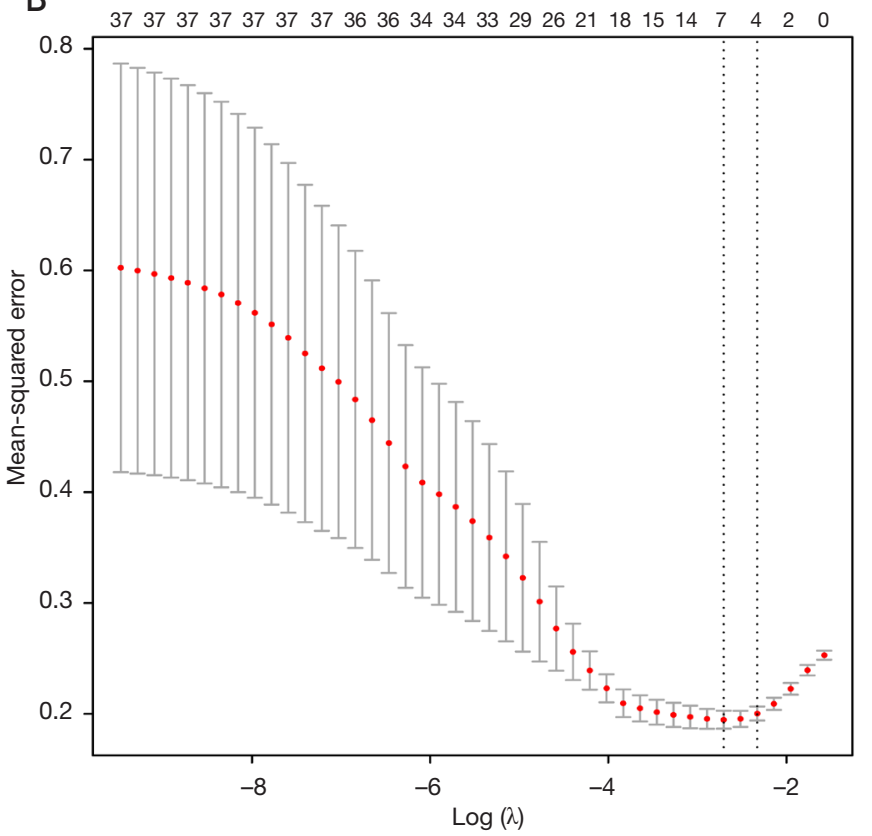

Figure 1 Screening of predictive factors. We used the LASSO regression method. (A) The LASSO regression method was used to choose predictive factors. (B) The penalty coefficient in the LASSO model was adjusted using cross-validation and minimum criteria. The vertical black line represented the optimal $\lambda$ (i.e., the model provided the best fit to the data). The minimum $\lambda$ was 0.080700 , and $\ln (\lambda)=-2.517017$. LASSO, the least absolute shrinkage and selection operator.

Table 2 Multivariate logistic regression analysis of the influencing factors screened by LASSO regression

\begin{tabular}{ll}
\hline Variable & P value \\
\hline cT stage before INJT & 0.0145 \\
CN stage before INJT & 0.0951 \\
CT stage after INJT & 0.0045 \\
WBCs before INJT & 0.0094 \\
Change in albumin & 0.0011 \\
\hline
\end{tabular}

LASSO, the least absolute shrinkage and selection operator; $\mathrm{CT}$, clinical T stage; INJT, immune neoadjuvant therapy; cN, clinical $\mathrm{N}$ stage; WBC, white blood cell.

immunotherapy. The receiver operating characteristic curve of this model is shown in Figure 3B. We also plotted the decision curve (Figure $3 C$ ) and the clinical impact curve (Figure $3 D$ ) for this model to evaluate the prediction model.

\section{Discussion}

In this study, we developed a prediction model to predict the likelihood of TRG 2-3 of esophageal cancer patients among those who underwent neoadjuvant chemotherapy combined with immunotherapy. Like other studies $(27,28)$, the results showed that the prediction model could well predict the TRG, and the model fit was high (Brier score 0.13 , calibration slope 0.99 , and C-index 0.88 ).

Neoadjuvant immunotherapy could ethically enhance the function of $\mathrm{T}$ cells and achieve better anti-tumor efficacy. Meanwhile, there will also be immune-related side effects, such as immune pneumonia, abnormal thyroid function, etc.

After reviewing the postoperative pathological results of the patients, we found that the patients with complete tumor shrinkage did not all have TRG $0-1$. There were 38 patients with complete clinical remission, but 18,12 , six, and two of them had TRGs of 0,1,2 and 3, respectively. For patients with TRG $0-1$, neoadjuvant chemotherapy combined with immunotherapy was likely to benefit patients and obviate the need for neoadjuvant radiotherapy treatment. This important benefit, neoadjuvant chemoradiotherapy may increase mortality $(7,8)$. However, for patients with TRG 2-3, neoadjuvant radiotherapy may be added to boost their survival. Therefore, we intend to screen these patients as candidates to receive neoadjuvant 

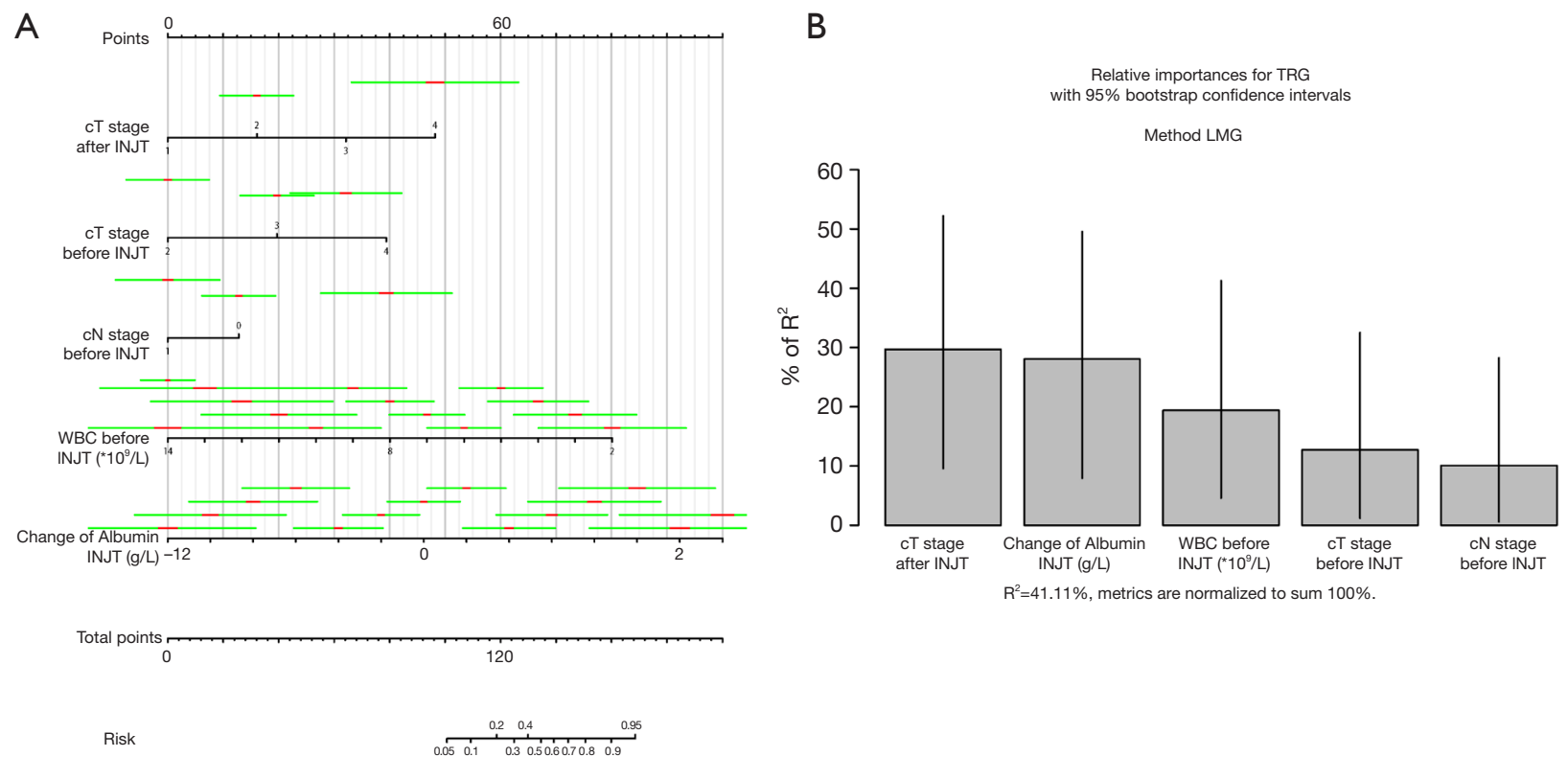

Figure 2 Nomogram plotted against various predictors and the importance of each predictor for TRG interpretation. (A) A nomogram for predicting the tumor regression grade of esophageal cancer patients treated with neoadjuvant immunotherapy. To use the nomogram, each factor has a score, and then the scores for each factor are added up to have a total score that corresponds to the likelihood of TRG 2-3 in the nomogram. (B) Degree of TRG variance explained by each influencing factor. INJT, immune neoadjuvant therapy; WBC, white blood cell; TRG, tumor regression grade; LMG, Lindeman, Merenda and Gold.

radiotherapy. However, it may not be accurate to rely solely on preoperative CT examination results to assess the need for combined radiotherapy. After all, in our study, $21.1 \%$ $(8 / 38)$ of the patients with complete clinical remission had a TRG of 2 or 3 . The prediction model we established can distinguish TRG 2-3 patients from TRG 0-1 patients well.

Our prediction model showed that the difference between the pre-neoadjuvant and preoperative albumin levels was correlated with the score, i.e., the greater the increase in albumin level after chemotherapy, the more likely the TRG was to be $2-3$. There are many studies on the relationship between albumin and the prognosis of solid tumors. (29-31). The albumin level is also associated with the prognosis of esophageal cancer $(32,33)$. However, these were all studies on how the ratios of albumin to other indices was correlated with prognosis. It was also found that patients with higher serum albumin $(>3.5 \mathrm{~g} / \mathrm{L})$ had a better prognosis (34). There are few reports on the correlation between the difference in albumin before $v s$. after neoadjuvant therapy and the prognosis or the TRG. In this study, we found that the changes in albumin from before to after chemotherapy were correlated with TRG, and the albumin difference explain much of the TRG variance
(Figure 2B), indicating that the nutritional level may have a great impact on the response to this combined therapy on our patients. Highlighting that nutritional support therapy is a particularly important part of clinical treatment of patients with esophageal cancer.

In our results, the WBC count before neoadjuvant therapy was also an independent predictor. The higher the WBC count, the higher the score, and the more likely TRG was $0-1$; otherwise, it tended to be TRG $2-3$. The WBC count is related to a variety of factors (infection, tumor, systemic inflammatory response, etc.) and can provide predictive information for a variety of diseases, such as cardiovascular diseases and type 2 diabetes. It is an important marker of the health status of the human body (35). Circulating tumor cells (CTCs) in the peripheral blood of cancer patients can form CTC-WBC clusters with immune cells, such as WBCs, which can promote the proliferation and metastasis of CTCs. Patients with CTC-WBC clusters found in peripheral blood may have a poor prognosis $(36,37)$. Since WBCs can form CTC-WBC clusters with tumor cells and these clusters are correlated with prognosis, our results, in which WBC count was a factor in the prediction model, are consistent with the above results. 

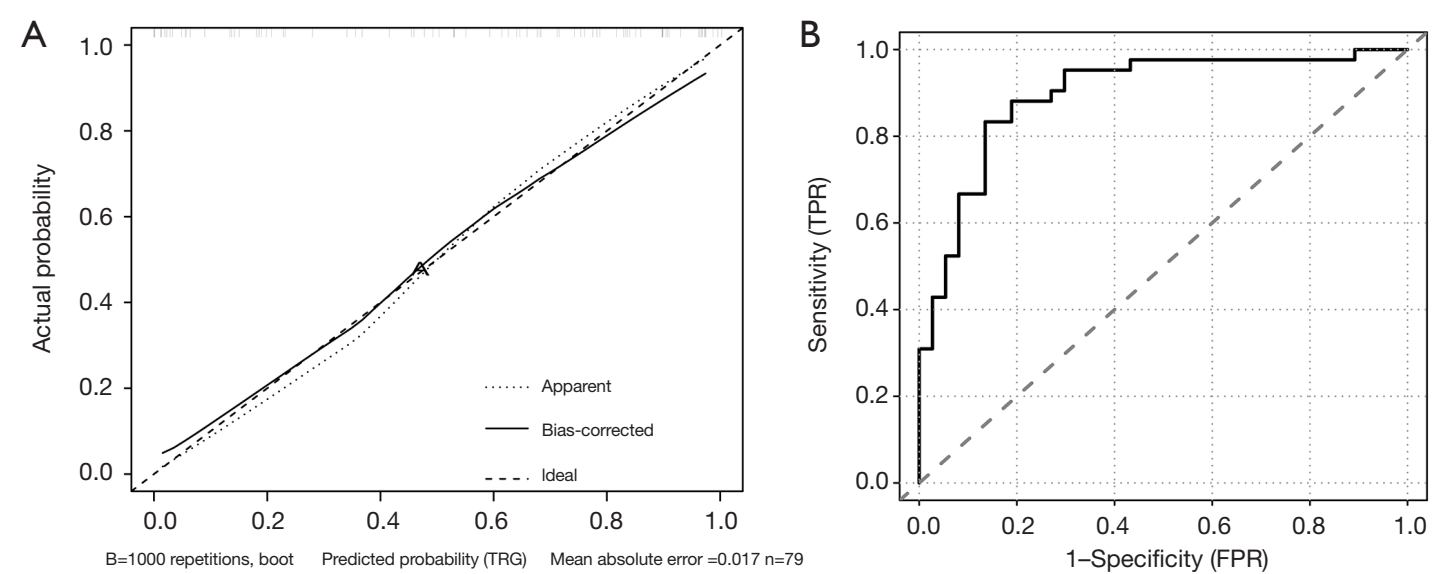

C

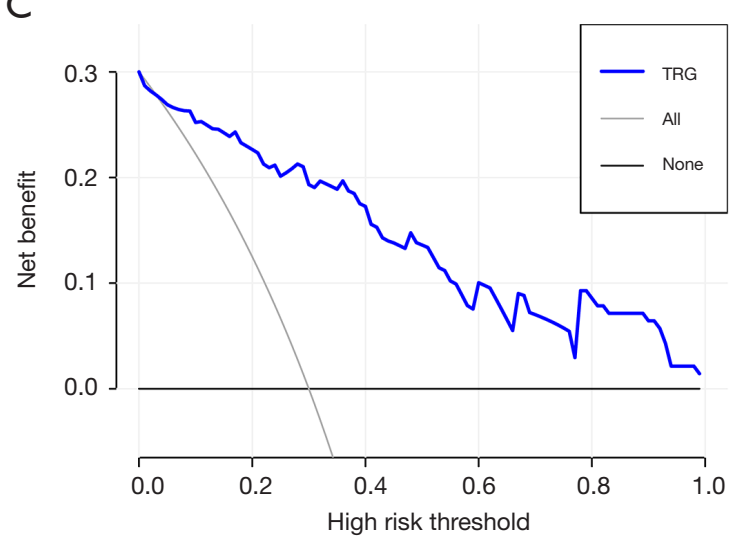

D

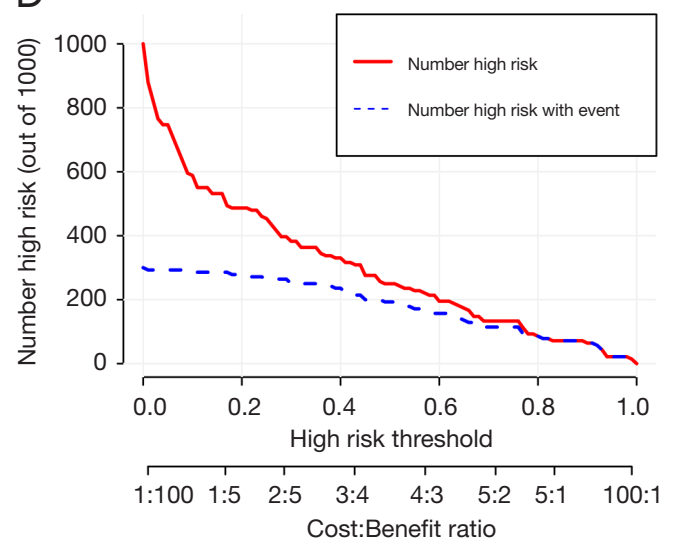

Figure 3 Various evaluation indicators of the prediction model (A) Calibration slope and (B) receiver operating characteristic curve of our model. Our model had a calibration slope of 0.98 and a C-index of 0.88. (C) Decision curve of the training cohort of the TRG 2-3 nomogram. (D) Clinical impact curve of the training cohort of the TRG 2-3 nomogram. TRG, tumor regression grade; FPR, false positive rate; TPR, true positive rate.

Among all patients, 39 of them underwent immunotherapy without PD-1 testing, and 40 patients underwent PD-1 testing, of whom 31 patients were positive. There are many studies on immunotherapy for esophageal cancer $(19,20,38)$. For patients with positive PD-L1, the response rate was higher than those with negative PD-L1 (ORR: 15.5\% vs. $6.4 \%$ ). For patients with unclear PD-L1, the response rate was in between the two (11.6\%) (39). Possibly because there was a high level of censored data $(39 / 79,49.4 \%)$, the PD-L1 status was not a factor associated with the TRG, so our prediction model did not include this factor. With the accumulation of more data, we plan to establish a predictive model for PD-L1-positive patients alone, and the results at that time may be more accurate and reliable for evaluating the effect of PD-L1 status on TRG in patients who have undergone neoadjuvant chemotherapy combined with immunotherapy.

Neoadjuvant immunotherapy could ethically enhance the function of $\mathrm{T}$ cells and achieve better anti-tumor efficacy. Meanwhile, there will also be immune-related side effects, such as immune pneumonia, abnormal thyroid function, etc. I think this Immune Neoadjuvant Immunotherapy could be used for other cancers, but some clinical researches need to be done.

We recognize that this study has limitations: (I) node positive patients with high scores before immune neoadjuvant therapy were more likely to have TRG $0-1$. It is difficult to explain this finding based on current knowledge, but we plan to examine it in a future study. (II) The sample of 79 patients was small, and a larger sample would yield better predictive value. (III) We did not include PD-L1 expression as an influencing factor in this study due 
to missing PD-L1 expression results. In the future, patients with PD-L1 test results will be included in the prediction model to determine the relationship between the efficacy of immunotherapy and PD-L1 expression in esophageal cancer.

\section{Conclusions}

Our prediction model may predict the likelihood of TRG $2-3$ in patients with esophageal cancer after immunotherapy combined with neoadjuvant chemotherapy. We plan to conduct a study of TRG 2-3 patients undergoing neoadjuvant radiotherapy using this prediction model.

\section{Acknowledgments}

The authors appreciate the academic support from the AME Esophageal Cancer Collaborative Group and AME for English language editing.

Funding: None.

\section{Footnote}

Reporting Checklist: The authors have completed the TRIPOD reporting checklist. Available at https://atm. amegroups.com/article/view/10.21037/atm-22-78/rc

Data Sharing Statement: Available at https://atm.amegroups. com/article/view/10.21037/atm-22-78/dss

Conflicts of Interest: All authors have completed the ICMJE uniform disclosure form (available at https://atm.amegroups. com/article/view/10.21037/atm-22-78/coif). JMP reports leadership or fiduciary role in Florida Chapter American College of Surgeons and Scientific Board ADVOCARE. The other authors have no conflicts of interest to declare.

Ethical Statement: The authors are accountable for all aspects of the work in ensuring that questions related to the accuracy or integrity of any part of the work are appropriately investigated and resolved. The study was conducted in accordance with the Declaration of Helsinki (as revised in 2013). This study was approved by the Ethics Committee of The Affiliated Cancer Hospital of Zhengzhou University (ethics number 2019092702), and informed consent was taken from all individual participants.

Open Access Statement: This is an Open Access article distributed in accordance with the Creative Commons Attribution-NonCommercial-NoDerivs 4.0 International License (CC BY-NC-ND 4.0), which permits the noncommercial replication and distribution of the article with the strict proviso that no changes or edits are made and the original work is properly cited (including links to both the formal publication through the relevant DOI and the license). See: https://creativecommons.org/licenses/by-nc-nd/4.0/.

\section{References}

1. Uhlenhopp DJ, Then EO, Sunkara T, et al. Epidemiology of esophageal cancer: update in global trends, etiology and risk factors. Clin J Gastroenterol 2020;13:1010-21.

2. van Hagen P, Hulshof MC, van Lanschot JJ, et al. Preoperative chemoradiotherapy for esophageal or junctional cancer. N Engl J Med 2012;366:2074-84.

3. Yang H, Liu H, Chen Y, et al. Neoadjuvant Chemoradiotherapy Followed by Surgery Versus Surgery Alone for Locally Advanced Squamous Cell Carcinoma of the Esophagus (NEOCRTEC5010): A Phase III Multicenter, Randomized, Open-Label Clinical Trial. J Clin Oncol 2018;36:2796-803.

4. Shapiro J, van Lanschot JJB, Hulshof M, et al. Neoadjuvant chemoradiotherapy plus surgery versus surgery alone for oesophageal or junctional cancer (CROSS): long-term results of a randomised controlled trial. Lancet Oncol 2015;16:1090-8.

5. Burmeister BH, Smithers BM, Gebski V, et al. Surgery alone versus chemoradiotherapy followed by surgery for resectable cancer of the oesophagus: a randomised controlled phase III trial. Lancet Oncol 2005;6:659-68.

6. Gebski V, Burmeister B, Smithers BM, et al. Survival benefits from neoadjuvant chemoradiotherapy or chemotherapy in oesophageal carcinoma: a meta-analysis. Lancet Oncol 2007;8:226-34.

7. Mariette C, Dahan L, Mornex F, et al. Surgery alone versus chemoradiotherapy followed by surgery for stage I and II esophageal cancer: final analysis of randomized controlled phase III trial FFCD 9901. J Clin Oncol 2014;32:2416-22.

8. Chan KKW, Saluja R, Delos Santos K, et al. Neoadjuvant treatments for locally advanced, resectable esophageal cancer: A network meta-analysis. Int J Cancer 2018;143:430-7.

9. von Dobeln GA, Klevebro F, Jacobsen AB, et al. Neoadjuvant chemotherapy versus neoadjuvant chemoradiotherapy for cancer of the esophagus or 
gastroesophageal junction: long-term results of a randomized clinical trial. Dis Esophagus 2019. doi: 10.1093/dote/doy078.

10. Klevebro F, Alexandersson von Döbeln G, Wang N, et al. A randomized clinical trial of neoadjuvant chemotherapy versus neoadjuvant chemoradiotherapy for cancer of the oesophagus or gastro-oesophageal junction. Ann Oncol 2016;27:660-7.

11. Sjoquist KM, Burmeister BH, Smithers BM, et al. Survival after neoadjuvant chemotherapy or chemoradiotherapy for resectable oesophageal carcinoma: an updated metaanalysis. Lancet Oncol 2011;12:681-92.

12. Kelsen DP, Winter KA, Gunderson LL, et al. Longterm results of RTOG trial 8911 (USA Intergroup 113): a random assignment trial comparison of chemotherapy followed by surgery compared with surgery alone for esophageal cancer. J Clin Oncol 2007;25:3719-25.

13. Allum WH, Stenning SP, Bancewicz J, et al. Long-term results of a randomized trial of surgery with or without preoperative chemotherapy in esophageal cancer. J Clin Oncol 2009;27:5062-7.

14. Boonstra JJ, Kok TC, Wijnhoven BP, et al. Chemotherapy followed by surgery versus surgery alone in patients with resectable oesophageal squamous cell carcinoma: longterm results of a randomized controlled trial. BMC Cancer 2011;11:181.

15. Urba SG, Orringer MB, Turrisi A, et al. Randomized trial of preoperative chemoradiation versus surgery alone in patients with locoregional esophageal carcinoma. J Clin Oncol 2001;19:305-13.

16. Weber JS, D'Angelo SP, Minor D, et al. Nivolumab versus chemotherapy in patients with advanced melanoma who progressed after anti-CTLA-4 treatment (CheckMate 037): a randomised, controlled, open-label, phase 3 trial. Lancet Oncol 2015;16:375-84.

17. Motzer RJ, Escudier B, McDermott DF, et al. Nivolumab versus Everolimus in Advanced Renal-Cell Carcinoma. N Engl J Med 2015;373:1803-13.

18. Reck M, Rodríguez-Abreu D, Robinson AG, et al. Pembrolizumab versus Chemotherapy for PD-L1Positive Non-Small-Cell Lung Cancer. N Engl J Med 2016;375:1823-33.

19. Hong Y, Ding ZY. PD-1 Inhibitors in the Advanced Esophageal Cancer. Front Pharmacol 2019;10:1418.

20. Shah MA, Kojima T, Hochhauser D, et al. Efficacy and Safety of Pembrolizumab for Heavily Pretreated Patients With Advanced, Metastatic Adenocarcinoma or Squamous Cell Carcinoma of the Esophagus: The Phase 2
KEYNOTE-180 Study. JAMA Oncol 2019;5:546-50.

21. Chao YK, Chang CB, Chuang WY, et al. Correlation Between Tumor Regression Grade and Clinicopathological Parameters in Patients With Squamous Cell Carcinoma of the Esophagus Who Received Neoadjuvant Chemoradiotherapy. Medicine (Baltimore) 2015;94:e1407.

22. Powell AGMT, Chin C, Coxon AH, et al. Neutrophil to lymphocyte ratio as a predictor of response to neoadjuvant chemotherapy and survival in oesophageal adenocarcinoma. BJS Open 2020;4:416-23.

23. Hermann RM, Horstmann O, Haller F, et al. Histomorphological tumor regression grading of esophageal carcinoma after neoadjuvant radiochemotherapy: which score to use? Dis Esophagus 2006;19:329-34.

24. Qiu MJ, Yang SL, Wang MM, et al. Prognostic evaluation of esophageal cancer patients with stages I-III. Aging (Albany NY) 2020;12:14736-53.

25. Takeda FR, Tustumi F, de Almeida Obregon C, et al. Prognostic Value of Tumor Regression Grade Based on Ryan Score in Squamous Cell Carcinoma and Adenocarcinoma of Esophagus. Ann Surg Oncol 2020;27:1241-7.

26. Iasonos A, Schrag D, Raj GV, et al. How to build and interpret a nomogram for cancer prognosis. J Clin Oncol 2008;26:1364-70.

27. Lemini R, Díaz Vico T, Trumbull DA, et al. Prognostic models for stage I-III esophageal cancer: a comparison between existing calculators. J Gastrointest Oncol 2021;12:1963-72.

28. Gabriel E, Attwood K, Shah R, et al. Novel Calculator to Estimate Overall Survival Benefit from Neoadjuvant Chemoradiation in Patients with Esophageal Adenocarcinoma. J Am Coll Surg 2017;224:884-894.e1.

29. Wang Z, Zhang L, Wang J, et al. Prealbumin-to-Globulin Ratio Can Predict the Chemotherapy Outcomes and Prognosis of Patients with Gastric Cancer Receiving FirstLine Chemotherapy. J Immunol Res 2020;2020:6813176.

30. Nie M, Sun P, Chen C, et al. Albumin-to-Alkaline Phosphatase Ratio: A Novel Prognostic Index of Overall Survival in Cisplatin-based Chemotherapy-treated Patients with Metastatic Nasopharyngeal Carcinoma. J Cancer 2017;8:809-15.

31. Yamamoto T, Kawada K, Hida K, et al. Combination of lymphocyte count and albumin concentration as a new prognostic biomarker for rectal cancer. Sci Rep 2021;11:5027.

32. Hu SJ, Zhao XK, Song X, et al. Preoperative maximal 
voluntary ventilation, hemoglobin, albumin, lymphocytes and platelets predict postoperative survival in esophageal squamous cell carcinoma. World J Gastroenterol 2021;27:321-35.

33. Tan Z, Zhang M, Han Q, et al. A novel blood tool of cancer prognosis in esophageal squamous cell carcinoma: the Fibrinogen/Albumin Ratio. J Cancer 2017;8:1025-9.

34. Gupta D, Lis CG. Pretreatment serum albumin as a predictor of cancer survival: a systematic review of the epidemiological literature. Nutr J 2010;9:69.

35. Zhang Y, Bai J, Wu H, et al. Trapping cells in paper for white blood cell count. Biosens Bioelectron 2015;69:121-7.

36. Luo Q, Wang C, Peng B, et al. Circulating Tumor-CellAssociated White Blood Cell Clusters in Peripheral Blood
Indicate Poor Prognosis in Patients With Hepatocellular Carcinoma. Front Oncol 2020;10:1758.

37. Sarioglu AF, Aceto N, Kojic N, et al. A microfluidic device for label-free, physical capture of circulating tumor cell clusters. Nat Methods 2015;12:685-91.

38. Kojima T, Shah MA, Muro K, et al. Randomized Phase III KEYNOTE-181 Study of Pembrolizumab Versus Chemotherapy in Advanced Esophageal Cancer. J Clin Oncol 2020;38:4138-48.

39. Fuchs CS, Doi T, Jang RW, et al. Safety and Efficacy of Pembrolizumab Monotherapy in Patients With Previously Treated Advanced Gastric and Gastroesophageal Junction Cancer: Phase 2 Clinical KEYNOTE-059 Trial. JAMA Oncol 2018;4:e180013.
Cite this article as: $\mathrm{Yu} \mathrm{Y,} \mathrm{Wang} \mathrm{W}$, Qin $\mathrm{Z}$, Li H, Liu Q, Ma H, Sun H, Bauer TL, Pimiento JM, Gabriel E, Birdas T, Li Y, Xing W. A clinical nomogram for predicting tumor regression grade in esophageal squamous-cell carcinoma treated with immune neoadjuvant immunotherapy. Ann Transl Med 2022;10(2):102. doi: 10.21037/atm-22-78 